\title{
YI.
}

\section{Platon und Aristoteles bei Apollinarios.}

Von

Dr. Johannes Dräseke in Wandsbeck.

Wenn ich seit einem Jahrzehnt bemüht gewesen bin, die schriftstellerische Persönlichkeit des Apollinarios von Laodicea aus dem Wust und den Trümmern einer Jahrhunderte lang irregeleiteten Ueberlieferung zu neuem Leben zu erwecken, so habe ich die Freude gehabt, die Ergebnisse meiner Forschungen allseitig angenommen, Apollinarios selbst von hervorragenden Fachgenossen als den grōssten Kirchenlehrer des 4. Jahrhunderts anerkannt zu sehen ${ }^{3}$ ). Auch auf die dichterische Bedeutung des Mannes habe ich aufmerksam zu machen gesucht und den Philologen, welche, wie Rzach und Ludwich, von einer Schule des Epikers Nonnos redend, gleichwohl anerkennen, „dass Apollinarios sich an die strenge Observanz des Meisters, namentlich in metrischen Dingen, durchaus nicht gebunden hat ${ }^{\alpha 2}$ ), die Möglichkeit entgegengehalten, dass auch gerade das Umgekehrte das Richtige sein könne, so zwar, dass Apollinarios, der bis 390 lebte und seine dichterische Thatigkeit in den sechziger und siebziger Jahren des 4. Jabrhunderts entfaltete. früher als Nonnos oder gleichzeitig mit diesem dichtete und sich auch in dichterischen Dingen ebenso eine lobenswerthe Lnabhängigkeit und Selbständigkeit wahrte, wie er das besonders in philosophischen Dingen gethan hat ${ }^{2}$ ). Für letztere Frage fehlt es noch an dem nöthigen Nachweis. Ihn will ich im Folgenden in kurzen Zügen zu geben versuchen.

3) II arnack, Lehrbuch der Dograengeschichte II, S. 312 fr.

$\Rightarrow$ A. Ludwich im, Ilermes" XIII, 1578, S. 347.

$\rightarrow$ Zeitschrift 2 . wiss. Theologie XXXI, S.479. 480 . 
Die in unseren Tagen der hohen Bedeutung des Apollinarios als Kirchenlehrer gezollte Anerkennung ist - allerdings eine merkwürdige Erscheinung - nichts weiter als eine Wiederholung desjenigen Urtheils, das schon sein Zeitgenosse Philostorgios über ihn fïllte. Mag dies immerhin als das eines Arianers - sicherlich mit Unrecht - bis auf unsere Tage angezweifelt worden sein, so wird man doch nicht in Abrede stellen dürfen, dass es durch einen anderen Zeitgenossen, den christlichen Philosophen $\mathbf{N}$ emesios jedenfalls in sehr gewichtiger Weise bestätigt wird. Denn von allen theologisch so bedeutenden Zeitgenossen nennt derselbe als Männer der Wissenschaft nur Eunomios und Apollinarios, den Bischof von Laodicea, ersteren an nur einer Stelle, letzteren an dreien ${ }^{4}$ ). Auch Suidas bezeugt das Gleiche, wenn er von

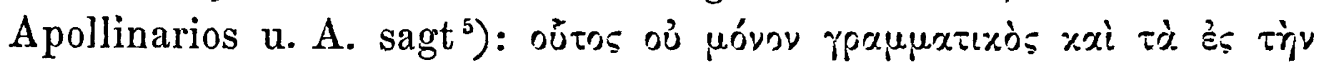

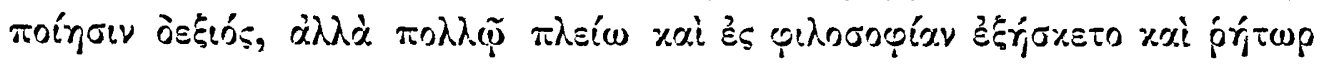

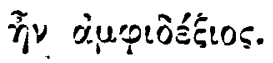

So lange man bei Apollinarios nur nach den dürftigen Bruchstücken seiner Schriften, die aus dem Alterthum uns überliefert sind, zu urtheilen genöthigt war, herrschte die besonders auf seine in der von Gregorios von Nyssa bekämpften christologischen

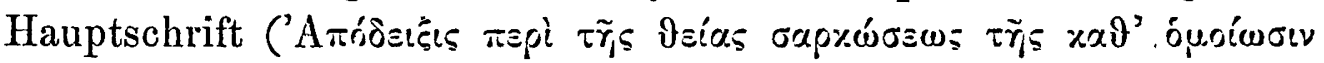
¿̀vopóño) hinsichtlich der Bezeichnung der Bestandtheile des Men-

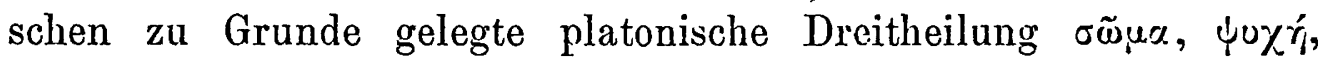
$\pi v \varepsilon \tilde{u} \mu \alpha$ gestützte Annahme, er sei Platoniker gewesen. Diese Annahme ist jedoch entschieden als eine ganz einseitige $z u$ bezeichnen. Das tritt insbesondere klar und einleuchtend hervor, wenn wir auf die Art und Weise achten, wie diese Beobachtung gelegentlich verwerthet worden ist. Wenn \%. B. H. Kihn in seinem "Theodor von Mopsuestia" (Freiburg 1880) Apollinarios wegen der von ihm "vorgetragenen platonisch-plotinischen Trichotomie" zum Platoniker stempelt, um den Gegensatz zu Theodoros von Mopsuhestia, der (S. 186). ,in seiner Christologie

4) Nemesius Emesenus, De natura hominis er. IIatthäi c. I, S. 36; c. II, S. 108.109 ; c. V, S. 166 .

5) Suidae Lexicon ed. Bernhardy I, S. 615. 
dem aristotelischen Begriffe von Natur und Person gefolgt ist", deutlich hervortreten zu lassen, so ist dies schief und der Sache nicht entsprechend. Dieses Einzwängen in das Muster und Vorbild der philosophischen Schulsprache, dies Aburtheilen und Einordnen nach den Schlagworten "Platoniker" und "Aristoteliker" ist durch-" aus irrelcitend und wird der. Fülle der besonders in Apollinarios sich zeigenden umfassenden Geistesbildung und der Mannigfaltigkeit der von ihm gehandhabten philosophischen Beweismittel in keiner Weise gerecht. Dieser Mangel tritt u. a. schon in dem vierten Kapitel seines Werkes hervor, in welchem Kihn eine allgemeine Schilderung der alexandrinischen und antiochenischen Schule giebt. „Bei den Alexandrinern", heisst es dort S. 7, "wog die nach geistiger Intuition des Göttlichen strebende Contemplation (Izwoix), der Ilealismus, die Speculation und Mystik vor; bei den Antiochenern war die nüchterne Verstandesrichtung, die logische Reflexion, die praktische Tendenz, die realistische Betrachtung der geoffenbarten Dinge (iఠoopíx) der eigenthümliche Charakterzug. Zur Ausbildung dieser beiderseitigen Geistesrichtung trug nicht wenig die. Verschiedenheit der philosophischen Systeme bei, denen sie huldigten. Während der zur Zeit der Grundlegung des Christenthums herrschende Eklekticismus in der Philosophie in beide Schulen Eingang fand, schlossen sich doch die Alexandriner mit Vorliebe der platonischen Philosophie und zwar in der Form des Neuplatonismus an, wie ihn heidnische Lehrer und der hellenistisch gebildete Jude Philo zur Geltung gebracht hatten; die Antiochener hingegen waren nach Theophilus, welcher Platoniker gewesen ist, dem Stoicismus und seit Paul von Samosata und Arius der aristotelischen Philosophie mit Vorliebe zugethan, deren scharfe Dialetik ihrem Geiste besonders zusagte und im Kampfe für die Wahrheit siegreiche Waffen verlieh." Schon Dorner's Bemerkungen gegen Ba ur hätten Kihn von dem Versuche abhalten sollen, in dieser rein äusserlich die Thatsachen ordnenden Weise jenes reiche und vielge-taltige wisenschaftliche Leben begreifen zu wollen. Jorner ${ }^{6}$ )

G Dorner, Entwickelungsgeschichte der Lehre von der P'erson Christi 1, S. Ais. Aun. ?. 
erklärt es für durchans verfehlt, die Kirchenlehrer auf den Platonismus zurückzuführen, die Arianer dagegen auf aristotelische Philosophie: aristotelisch Gebildete, betont er mit Recht, finden sich auch unter den Kirchenlehrern, und weder die Lehre von der Schöpfung, noch rom Sohne, noch auch von Gott bei Eunomios sind aristotelisch. Bei aller Anerkennung der Thatsache der in dem gemeinsamen empirischen Grundzuge liegenden Verwandtschaft der Arianer mit Aristoteles und der Bildung derselben durch dessen Dialektik, hält es Dorner „mehr für verwirrend als förderlich, für spielend aber nicht den Inhalt beachtend, wenn man mit Baur schliesslich die Arianer und ihre kirchlichen Gegner in Aristoteliker und Platoniker eintheilen will." An den bciden dem Alexandriner Athanasios so nahe stehenden Kirchenlehrern Gregorios von Nyssa und Apollinarios von Laodicea zeigt sich das Unhaltbare jenes äusserlichen Verfahrens deutlich. Gregorios fühlte sich stark von Aristoteles angezogen, von dessen eindringender, wiederholter Durchforschung viele seiner Schriften zeugen, während er andererseits dem gewandten aristotelischen Dialektiker Eunomios .gegenüber, um zu beweisen, dass drei Götter auch ein Gott seien, und ein Gott wiederum zu dreien werde, sich der platonischen Anschauungs- und Ausdrucksweise bedient, so zwar, dass er die Philosophie Platon's nicht als die nothwendige Form der Wahrheit betrachtete, für deren Vertheidigung er einzustehen habe, sondern wechselnd mit den Waffen, platonischen wie aristotelischen, je nachdem die Stellung des Gegners es erforderlich erscheinen liess. Ganz ebenso liegt die Sache bei Apollinarios, in dessen Unterscheidung von voũs und $\psi v \times \dot{\eta}$ sowohl, als darin, dass an die Stelle des voũs das Gleichartige, eine höhere Potenz, getreten sei, Baumgarten-Crusius zwiefachen Platonismus fand ${ }^{7}$ ).

Seitdem wir nunmehr durch Caspari's' ${ }^{8}$ ) und meine eigenen Bemühungen in einen weiten Kreis von Schriften des Laodiceners klaren Einblick gewonnen haben, können wir 'über seine philo-

7) Baumgarten-Crusius, Lehrbuch der christl. Dogmengesch. II, S. 160.

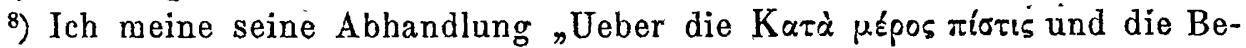
kenntnisse in ihr" in der Schrift „Alte und neue Quellen zur Geschichte des Taufsymbols und der Glaubensregel" (Christiania 1879), S. 65-146. 
sophische Stellung und Abhängigkeit, besonders von Platon und Aristoteles zutreffender als bisher urtheilen. In erster Linic sind wir dazu durch den bisher unter Justinus' des Märtyrers

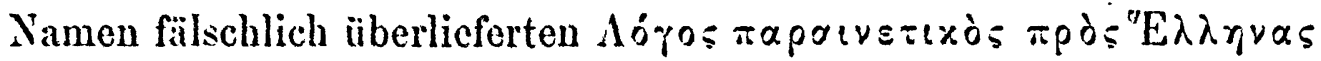
in den Stand gesetzt, den ich als dasjenige Werk des Apollinarios ron Laodicea nachgewiesen habe, welches er im Jahro 362 in Folge des rom Kaiser gegen die christlichen Lehrer erlassenen feindseligen Gesetzes unter der Nebenaufschrift ' 1 ' $\pi \grave{\rho}$ $\left.\dot{x}\rangle, \gamma, 8 s i \alpha \varsigma^{9}\right)$ an die von Julianus zu neuem Kampfe gegen das die Welt siegreich überwindende Christenthum aufgerufenen Hellenen richtete. In seinem für die Geschichte der alten Philosophie grundlegenden Werke über die griechischen Doxographen hat II. Diels ${ }^{10}$ ) nachgewiesen, dass eine ganze Reihe späterer Schriftsteller, wio Theodoretos, Nemesios, Ps. $=$ Plutarchos, Stobäos, in ihren Mittheilungen über griechische Philosophen abhängig sind von des am Ende des ersten oder Anfang des zweiten Jahrhunderts (S. 101)

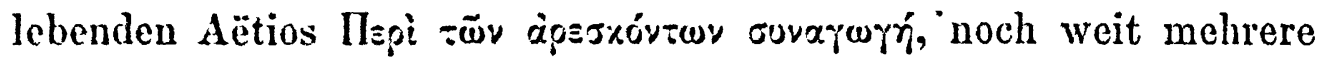
aber, wie Athenagoras, Eusebios, Kyrillos, Galenos, Laurentios Lydos. Ps. = Justinus, Achilles u. a., von dem in der Mitte des zweiten Jahrhunderts unter des Plutarchos Namen aus dem umfangreicheren Werke des Aëtios gefertigten Auszuge. Den Verfasser der Cohortatio führte Diels S. 17 unter den Ausschreibern des Plutarchischen Auszuges an letzter Stelle auf, weil auch ihm dessen Zeit nicht feststand. Wie Andere schon geurtheilt, schloss er aus der Thatsache, dass Kyrillos in seinem zweiten Buche gegen Kaiser Julianus (S. 48 B C) einige Stellen aus dem 6. und 7. Kapitel der Cohortatio fast genau wörtlich wiedergiebt, der Verfasser der letzteren müsse älter als $\mathrm{Ky}$ yrillos sein; vielleicht, meinte er, dürfte er dem Zeitalter des Athenagoras nicht fern stehen, mit dessen Art und Weise einer freicren Benutzung schriftlicher Vorlagen sowie dessen sonstiger Gelehrsamkeit er Verwandtschaft zeigt. Zum Erweise der schriftstellerischen Selbständigkeit des Verfasscrs der Cohortatio weist Diels darauf hin, dass er im 5. Kapitel die falsch-

") Snzum. Hint. eccle*. $v, 18$.

s) 11. Diels, Doxographi (iracci. Herolini, G. Reimer. 1879. 
lich dem A ristoteles beigelegte Schrift Ilepi «ígsov, welche jünger als Poseidonios ist, namentlich anfüht und im 7 : Kapitel die verschicdenen Ansichten über die Seele vielleicht unmittelbar aus

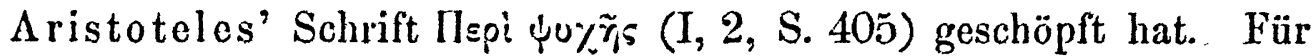
seine im 3. Kaipitel gegebenen Nachrichten aber über die alten Philosophen, zeigt Diels überzeugend, ist der Verfasser, wie auch v. Otto in seiner Ausgabe verzeichnet, ohne aus diesen Thatsachen irgend welchen Schluss zu ziehen, von dem Plutarchischen Aus-

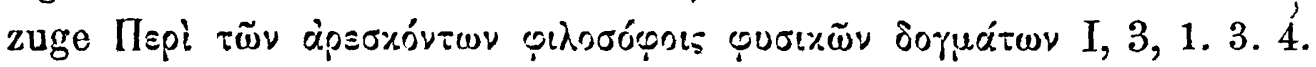
11. 5. 6. 7. 8. 18. 20 abhängig, so jedoch, dass er nicht allein die Reihenfolge geändert und Herakleitos unter den jonischen Philosophen aufführt, sondern auch in der Darstellung nach eigenem Ermessen sich Abweichungen erlaubt hat. Wenn der Verfasser im 5. Kapitel über Thales Ausführlicheres mittheilt, und Diels hervorhebt, dass von den übrigen in demselben Zusammenhange erwähnten philosophischen Ansichten sich nichts mit Sicherheit auf Plutarchos zurückführen lasse, so werden wir nicht fehl gehen, wenn wir darin einen Beweis für die umfassendere gelehrte Bildung des Verfassers erblicken, die eben Apollinarios in ganz besonderem Maasse zu Gebote stand. Die ganze Schrift ist ein lebendiges Zeugniss von der genauen. Vertrautheit des Laodiceners mit den Schriften und Lehren Platon's. Der zuerst wohl von Justinus dem Märtyrer ausgesprochene Gedanke von der Wirk-

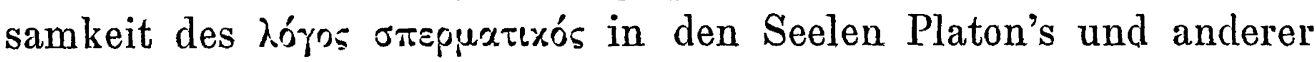
grosser hellenischer Geister vor Christus und ihrer Bekanntschaft mit dem Alten Testament, jener so gewinnende und bedeutungsvolle Gedanke, durch welchen das Christenthum als der gesammten vorchristlichen geistigen Entwickelung Abschluss erscheint, wiid hier von Apollinarios des Weiteren ausgeführt. Seinen Darlegungen zufolge (Kap. 20) hat Platon die Gotteslebre Moses' und der Propheten, welche er in Aegypten kennen lernte, angenommen, hat sie aber, aus Furcht vor dem Geschicke des Sokrates, für Gläubige und Ungläubige in seinen Schriften verschieden dargestellt ${ }^{11}$ ). Im Folgenden erörtert Apollinarios sodann die Unbe-

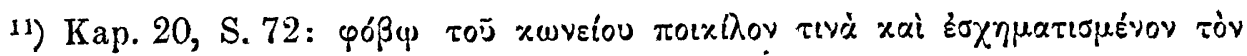


nennbarkeit Gottes und giebt cine Erklärung von dem Entstehen der ersten falschen Göttervorstellungen und von der Nothwendigkeit

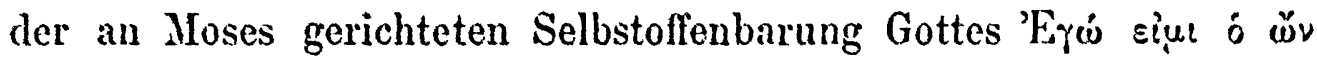
(Kap. 21). Alles dies - so führt er fort - lernte Platon in Aegypten, doch verschwieg er vor den Athenern den Namen des Moses. Seine Worte in Timïos, aus welchem Apollinarios die von Kirchenlehrern öfter benut\%to Stelle p. $27 \mathrm{D}, 28 \mathrm{~A}$ ed. Steph. wörtlich anführt, zeugen ihm ron dem Untergange der geschaffenen Götter. Besonders anziehend ist des Laodiceners Nachweis des Widerspruches, in welchen Platon durch seine verschiedenen Aussagen über die Gottheit und die Götter sich verwickelt (Kap. 23). Nach seiner Auffassung bat Platon durchaus keinen Grund, Homeros darob zu tadeln, dass er die Götter wandelbar nennt; und wenn er den Stoff, aus welchem die Götter geschaffen wurden, bald ungeschaffen, bald geschaffen nennt, so verfällt er in denselben Fehler, den er an Ilomeros rïgt. Offenbar hat Platon, so entschuldigt auch hier wieder Apollinarios den grossen Philosophen, aus Furcht vor len Verehrern der vielen Götter alsichtlich widersprechend über die Götter gelehrt. Was er aus Moses und den Propheten von einem Gotte überkommen, das hat er für die wahrhaftigen Gottesverehrer mystisch dargestellt. Ja in Platon's vicl angefübrtem

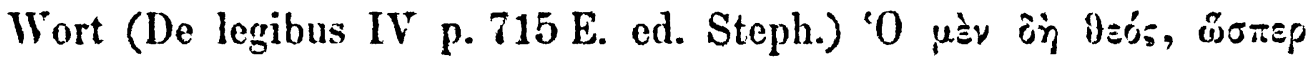

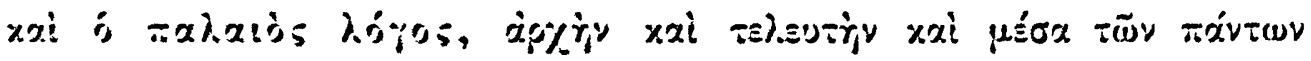
兰 (w) findet er eine unmittelbare Beziehung auf Moses' Gesetz, wohei er nur wieder den Namen des grossen Hebräers von Platon au; Furcht vor dem Schierlingsbecher verschwiegen sein lässt (hap. 25). Auch den Propheten hat Platon nach Apollinarios (Kap. 26) eine höhere und reinere Gotteserkenntniss zugeschrieben. Er führt zum Beweise dessen jene Stelle aus dem Timäos (p. $53 \mathrm{D}$ ) an. wo es von den Cranfïngen des Feuers und anderer irdischer Körper u. a. heirst: $-\dot{\alpha} ;$ j̇

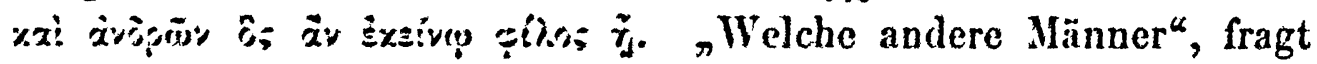
Apollinarios, „nennt er hier Freunde Gottes, als Moses und die

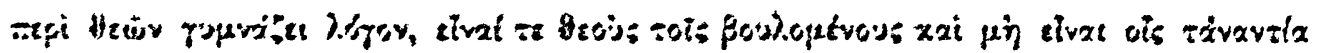

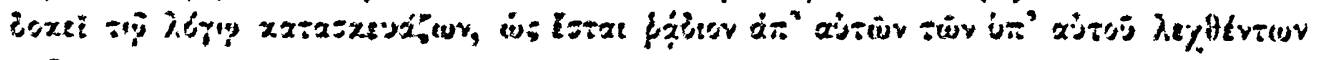
Troñose. 
Propheten?" Deren Schriften hatte Platon in Acgypten gelesen, aus ihnen entnahm er seine Lchre vom Endgericht. Das bestätigt ihm jene Stelle aus dem ersten Buche der Republik (p. 330 DE, $331 \mathrm{~A}$ ), welche er wörtlich mittheilt, nicht minder die Geschichte von Aridäios in der Unterwelt, die er aus dem zehnten Buche der Republik (p. 615 CDE, 616 AB) in seine Darstellung versetzt, um zu beweisen, dass Platon nicht allein die Lehre vom Gericht, sondern auch von der Auferstehung, an welche die Hellenen nicht glauben, von den Propheten überkommen hat ${ }^{12}$ ). Dass Apollinarios sogar Platon's Ideenlehre auf Moses (Kap. 29, S.,101), seine Lehre von dem geflügelten Wagen des Zeus auf den Propheten Ezechiel $(10,18.19)$ zurückführt, will ich nur im Vorübergehen erwähnen, um noch eine andere Beziehung seiner von gründlicher Kenntniss Platon's zeugenden Schrift hervorzuheben, die uns in den religiösen und zugleich philosophischen Geisterkampf jener Tage einen Blick thun lässt.

Apollinarios stand damals als der anerkannte Wortführer und Vorkämpfer der Christen auf dem Plane. Aus dem Eingang seines zweiten Briefes an Basileios geht dies deutlich hervor ${ }^{13}$ ). Unbedingt im Hinblick auf die ersten feindseligen Massregeln des Kaisers Julianus klagt er über den Kampf, der gegen die Frömmigkeit sich erhoben, er selbst stehe inmitten der Schlachtordnung und rufe die Freunde zum Beistande wider die Gewalt der Feinde auf. Das ist die Zeit, in welcher Apollinarios gegen den Kaiser oder die hellenischen Philosophen, wie Sozomenos sagt (V, 18:

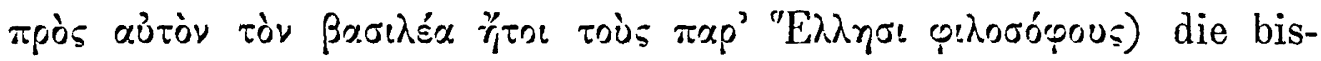
her behandelte Schrift, den $\Lambda$ ó ohne seinen Namen zu nennen. Wenn nun nach demselben Gewährsmann der Kaiser infolge der Schrift des Apollinarios in einem Schreiben an die hervorragendsten Bischöfe die Worte fallen liess

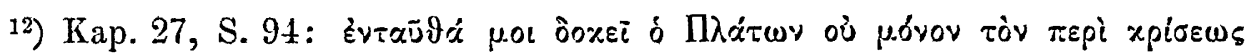

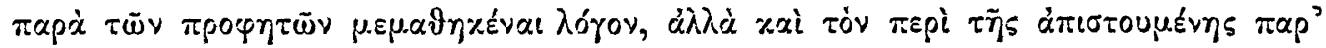

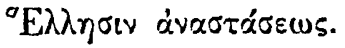

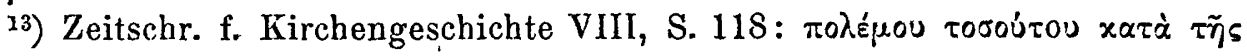

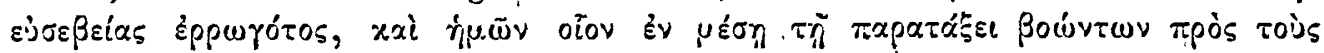

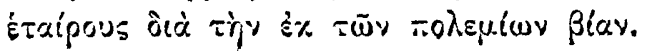




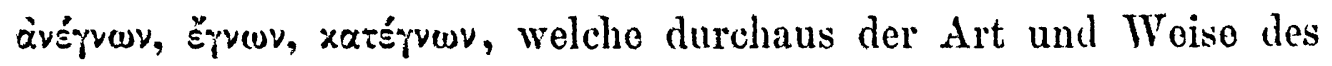
auf dio Wiederherstellung des Hellenismus eifrig bedachten Kaiscrs entsprechen: so liegt die Annahmo überaus nahe, dass Julianus, wenn er auch zunächst die christliche Schrift schroff zurïckwies,

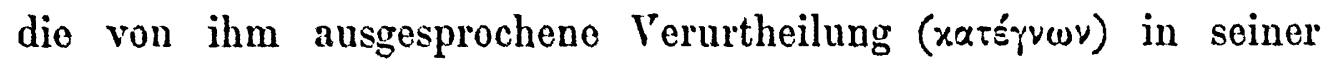
Schrift "Wider dic Christen", welche er in Winter 362 auf $363 \mathrm{zu}$ Antiochia ausarbeitete, werde wissenschaftlich gerechtfertigt, mit anderen Worten, dass er den cinzigen christlichen Gegner, der ihm bei seinen Lebzeiten mit einem Schriftwerk entgegentrat, werde wenn auch nicht Stück für Stück zu widerlegen gesucht, so doch in wichtigen Punkten berücksichtigt haben. Und in der That, wenn wir daraufhin das erste, in recht erfreulicher Vollständigkeit erhaltene Buch des Kaisers durchmustern, so ergeben sich die überraschendsten Vergleichspunkto, dio alle erst, wie mir scheint, durch Rückbeziehung auf die vorausgegangene Schrift des Apollinarios in das helle licht des vollen Verstïndnisses gerüclit werden.

Apullinarios hat aus hellenischen Quellen das hohe Alter des Moses nachgewiesen (Kap. 9), das weit über den Anfang hellenischen Schriftthums hinausreiche. Von ihm, dem aus Chaldäergeschlechte stammenden und in Aegypten in aller Weisheit von den Priestern Unterwiesenen sagt er nun (Kap. 10, p. $11 \mathrm{CD}$ ): ,Diesem verlieh Gott zuerst jene göttliche und prophetische Gabe, die damals heilige Männer von oben überkam, und rüstete ihn aus zum ersten Lehrer der Gottesverehrung, nach ihm sodann die ïbrigen Propheten, die gleich ihm derselben Gabe theilhaftig wurden und uns über ebendasselbe belehrt haben." Nachdem er so Moses: Ceberlegenheit und massgebende Bedeutung festgestellt, führt Apollinarios aus, wie Platon, der - was zuvor schon erwahnt wurde - in Aegypten des Moses Jchre kennen lernto (Kap. 20), von ihm in den wichtigsten Stücken der Lehre von Gott und Schöpfung abhängig sei, so zwar, dass er, durch Sokrates' Goschick geschreclit (Kap. 20), aus Furcht vor den Anhängern der Vielgötterei (Kap. 25), von Gott widersprechend gelehrt, seine wahre Meinung ror Linberufenen verhüllt, nur wahrhaft Gottesfürchtigen genügend angedeutet habe. Aus diesen, in einigen 
Stïcken schon vorher berührten, Sachverhalte wird es erst vollständig klar, warum Julianus gleich im ersten Buche seiner Schrift gleichfalls auf Moses'. Schöpfungsbericht zurückgreift, im Gegensatz zu Apollinarios aber so, dass er Platon's Ueberlegenheit in jeder Ilinsicht klarzulegen sich bemüht. „Man achte also darauf", sagt er (S. $49 \mathrm{~A}$, nach Neumann's Uebersetzung), "was dieser vom Schöpfer aussagt und was für Worte er ihm bei der Entstehung der Welt in den Mund legt, damit wir den Schöpfungsbericht des Platon und des Moses einander gegenüberstellen können. Dabei dürfte es wohl zu Tage treten, wer den Vorrang.verdient und in höherem Grade des Verkehrs mit der Gottheit würdig war, ob Platon, der den Götterbildern fromme Verehrung erwies, oder der Mann, von dem die Schrift sagt, dass mündlich Gott zu ihm geredet hat." Die in gesperrter Schrift ausgehobenen Worte weisen unverkennbar auf A pollinarios' Versuch zurück, des Moses entscheidendes Ansehn in erster Linie auf die ihm von Gott unmittelbar gewordene Geistesmittheilung und prophetische Begabung zu gründen.

Auf alle Einzelheiten der sorgfältigen Darstellung des Kaisers kann hier nicht eingegangen werden, ein Punkt aber aus dem Vergleich der Mosaischen und, Platonischen Schöpfungsgeschichte ist besonders auffüllig. Mit Nachdruck hat Apollinarios darauf aufmerksam gemacht, dass Platon in seinem Timäos über Gott dasselbe wie Moses lehre, ja dass er sogar im Ausdruck von ihm abhängig sei. Den von Platon im Timäos (p. $41 \mathrm{~A}$ ) ge-

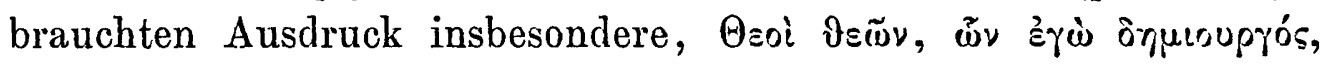
behandelt Apollinarios ausführlich (Coh. Kap. 20, n. 13; Kap. 22, n. 13; Kap. 24, n. 5). Er schliesst (p. 21 AB) aus der TimäosStelle, Platon habe den Ungewordenen zwar als ewig bezeichnet, klar aber von dem Werden und Vergehen der Götter geredet, und weist Kap. 23-25 auf die offenbaren. Widersprüche hin, welche sich aus der Darstellung Platon's (Tim. p. $27 \mathrm{D}-28 \mathrm{~A}, 41 \mathrm{~A} \mathrm{~B}$ ) ergeben. Apollinarios' prïfende Untersuchung ist da ungemein scharf und eindringend. Das scheint Julianus besonders tief empfunden zu haben, denn er widmet den von Apollinarios angezogenen Timäos-Stellen eine eingehende Betrachtung, düch welche 
er vor Allem den Schlussfolgerungen des Gegners sich entziehen zu wollen scheint. Er führt (S. 58 B ff.) aus dem Timïos zunächst p. $41 \mathrm{ABC}$ wörtlich an und fährt dann (S. $65 \mathrm{~A}$ ) fort: „Aber erwäget, ob dies nicht etwa eine Träumerei ist, und lasset es euch erklären. Als Götter bezeichnet Platon sichtbare Wesen wie Sonne und Mond, die Sterne und den Himmel; aber diese sind nur Abbilder von unsichtbaren. Dio unseren Augen erscheinende Sonne ist das Abbild einer geistigen und unsichtbaren, und ebonso ist der Mond, den unsere Augen erblicken, und jeder der Sterne das Abbild eines geistigen Wesens. Platon kennt nun diese unsichtbaren Gottheiten, die in und mit dem Schöpfer existiren und aus ihm durch Zeugung hervorgegangen sind. Angemessen sagt daher bei ihm der Schöpfer „Götter", wobei er sich an die unsichtbaren wendet, ,ron Göttern", nämlich den erscheinenden. Beide aber haben einen gemeinsamen Schöpfer, ihn, der Himmel und Erde, das Meer und die Sterne gebildet und jedem von ihnen ein Urbild in einem geistigen Wesen erzeugt hat." Nachdem Julianus dann ferner aus Aussprïchen der heiligen Schrift, aus Moses und den Propheten (auch diese Wortverbindung theilt er mit Apollinarios) erwiesen, dass man (S. $100 \mathrm{C}$ ) »den Gott der Juden nicht fïr den Schöpfer der ganzen Welt und den Herrn aller Dinge zu halten ${ }^{*}$ habe, sondern dass er „bei seiner begrenzten Herrschaft auf einer Stufe mit den übrigen Göttern gedacht werden" müsse, fragt er: „Sollen wir noch auf euch hören, da ihr, oder doch einer aus eurem Stamme, in curen Gedanken von dem Gotte des Alls bei einer mindestens dürftigen Vorstellung angelangt seid?" Diese Frage scheint unmittelbar gegen A pollin arios' Klage (Kap. 9, p.9D; hap. 11. p. $11 \mathrm{E}$; Kap. 38 , p. $36 \mathrm{D}$ ) gerichtet, dass dio Hellenen um des Wahnes ihrer Vorfahren willen Moses und den Propheten noch keinen Glauben schenken wollen. Ja wenn der Kaiser (S. $253 \mathrm{~B})$ von den Christen ausdrücklich sagt: „Sie erklären, vor Allem dem Moses und den Propheten zu folgen, welche nach ihm in Judäa aufgetreten sind ${ }^{\alpha}$, so erscheint dies unmittellar bezogen auf A pollinarios' Erklärung hinsichtlich der hohen Bedeutung des Mases und der übrigen "l'ropheten nach ihn"

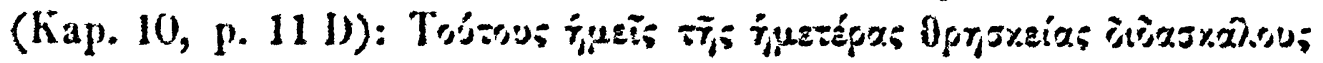




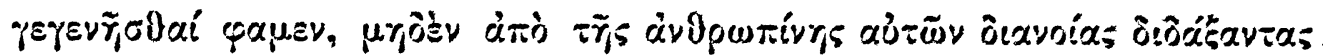

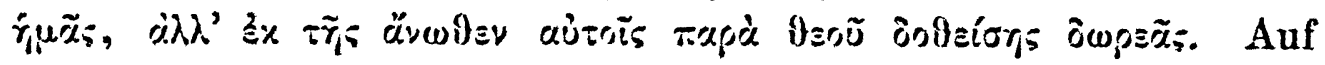
dieselbe Stelle scheint der Kaiser zuriickzublicken, wenn er im weitcren Verlauf der eben berührten Darlegungen entgegnet (S. 144C): „Aber sehet zu, ob Gott nicht vielleicht auch uns göttliche Leiter von hoher Trefflichkeit gegeben hat, von denén ihr keine Kunde hattet, die aber in nichts dem bei den Hebräern von Anfang an verehrten Gotte Judäas nachstehen, “ und zum Schlúss seiner Auseinandersetzungen die Aufforderung ergehen lässt (S.148C): "Wenn aber Moses einen Theilgott verehrt hat und die Herrschaft über das All in einem Gegensatz zu ihm steht, so ist es besser, wenn man uns folgt und den allwaltenden Gott erkennt." Ich schweige von weiteren, höchst überraschenden Einzelheiten, deren noch mehrere vorhanden sind. Jedenfalls stossen wir im ersten Buche des philosophischen Kaisers fort und fort auf Beziehungen, für welche die Darlegungen des Apollinarios mehr oder weniger deutlich die Voraussetzung bilden.

Haben wir so den Umfang der aus genauer Kenntniss Platon's unmittelbar geschöpften Lehren bei Apollinarios kennen gelernt, wobei ich die merkwürdige, von der Weltschöpfung handelnde und gleichfalls auf Platon zurückgehende Stelle des Apollinarios bei Nemesios (Kap. 5, S. 166) unberücksichtigt lasse; weil ich an andrem Orte über sie ausfübrlich geredet habe ${ }^{14}$ ), so tritt uns in den eigentlichen Lehrschriften des Laodiceners eine ungewohnte Fülle von Beziehungen in Sprache und Gedanken entgegen, die von vertrauter Bekanntschaft mit den Werken des Aristoteles und der Fähigkeit, selbständig die aus ihnen gewonnene Erkenntniss in der Ausgestaltung und Vertheidigung der christlichen Lehren zu verwerthen, worauf schon Diels in einer zuvor mitgetheilten

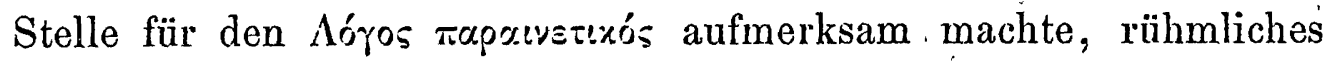
Zeugniss ablegen.

Sehen wir ihn, wie ich vorher bereits anführte, in seinem „Erweis der göttlichen Fleischwerdung nach dem Bilde des Menschen" von der platonischen Dreitheilung $\sigma \tilde{\omega} \mu \alpha, \downarrow \nu \chi \gamma^{\prime \prime}, \pi v \varepsilon \tilde{u} \mu \alpha$

14) Zeitschr. f. wissenschaftliche Theologie XXIX, S. $31 \mathrm{ff}$. 
aus, die er nur nachträglich oder beiläufig aus der Schrift begründete, das Wesen des Menschen bestimmen und christologisch dieselbe verwenden, so war er, der aristotelisch gründlich geschulte Denker geneigt, „über dem Besonderen und Unterscheidenden das Allgemeine und Identische $\mathrm{zu}$ vernachlässigen ${ }^{(15}$ ), und wenn Gregorios von Nyssa (Antirrhet. c. 35, p. 209) von ihm be-

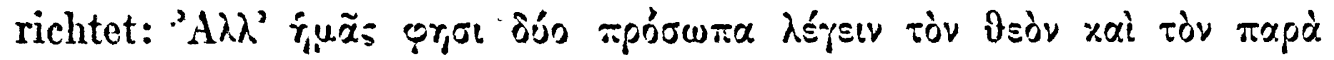

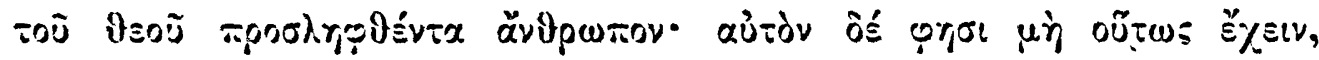

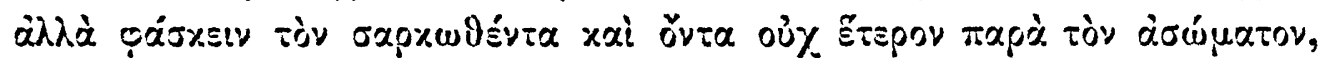

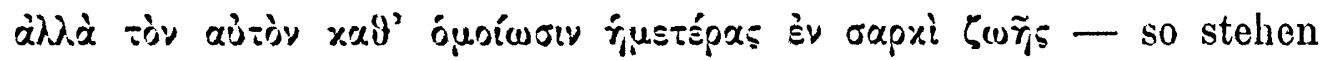
wir hier auf aristotelischem Boden, und das von Apollinarios in derselben Schrift angewandte dialektische Verfahren ist eben das des Aristoteles. Aristotelisch in der Fassung und im sprachlichen Ausdruck ist ferner auch die von ihm in der Kard

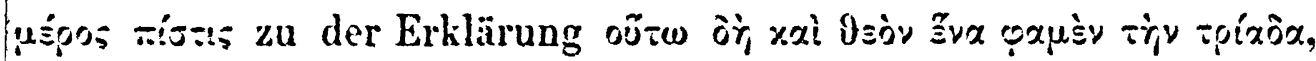

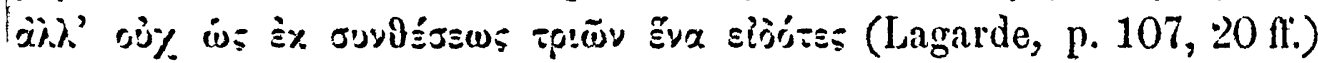

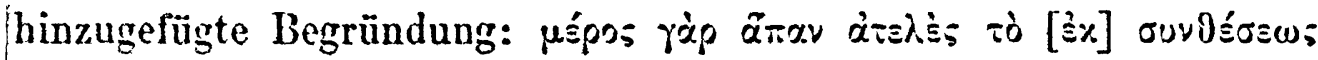
j:-5:xiszvo\%. Sie erinnert, um zum Vergleich nur ein Beispiel aus Aristoteles' Ethik anzuführen, an jene Stelle des zelinten Buches, wo der Philosoph, von der Lust redend, in folgender Weise seinen Gedanken durch ein Beispiel erläutert $(X, 3 ;$ p. 1174, 20=

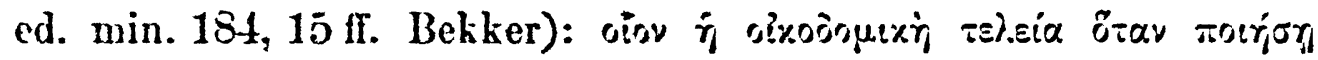

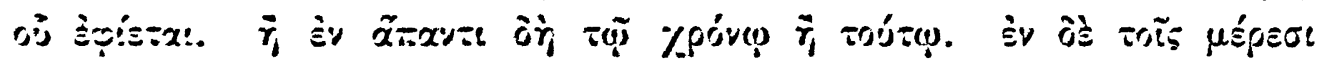
-jij $\mathrm{Zu}$ der Stelle des Apollinarios kann vielleicht auch Aristot. de

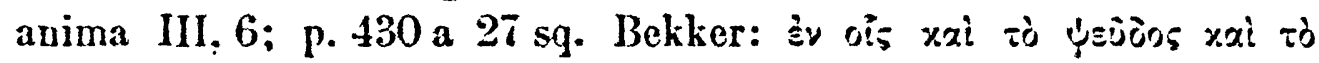

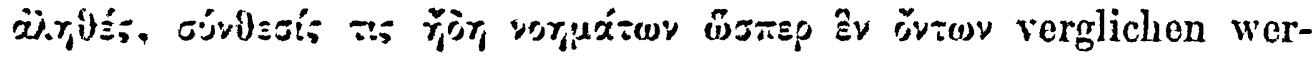
den. Ganz besonders aber mache ich auf jene von Kaiser Justinianus in seiner Schrift gegen die Monophysiten (Mai, Script. vet. nov. coll. VII, p. 310) uns aufbewahrte vortreffliche Stelle aus A pollinarios' Syllogismen aufmerksam, welche durch und durch aristotelisch

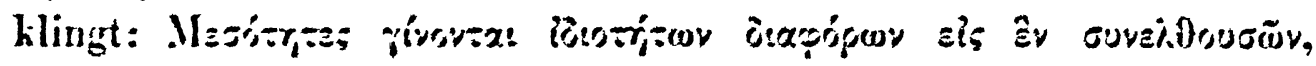

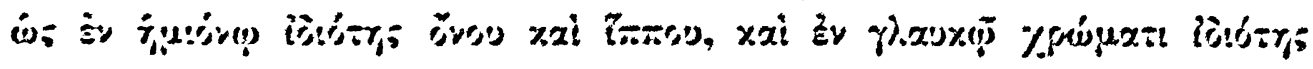

13) J. Rupp, Gregor's, des Bischofs ron Nyssa, Leben und Meinuigen (Leiprig 1834), S. 139. 


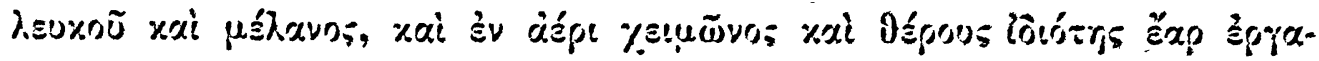

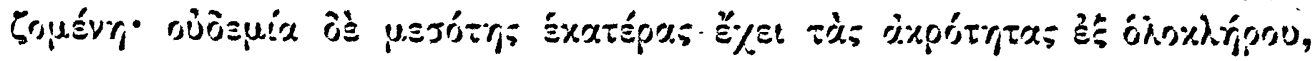

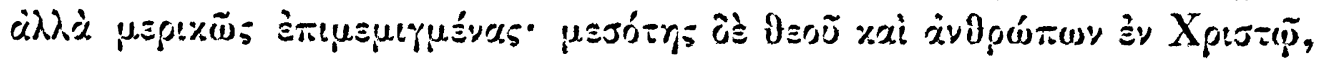

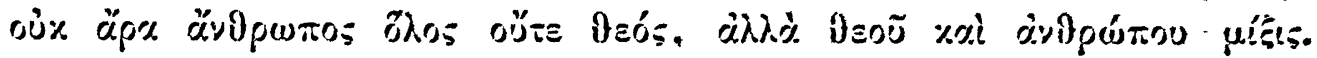
Hier vor Allem zeigt es sich, dass Apollinarios ein in erster Linie aristotelisch gebildeter Denker war, eine Thatsache, welche auch Harnack unumwunden anerkannt und gelegentlich besonders hervorgehoben hat. Die ganze Stelle erinnert auf das lebhafteste an des Aristoteles in seiner Nikomachischen Ethik so gründlich be-

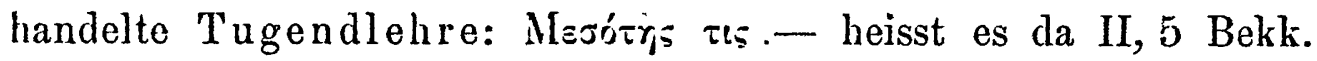

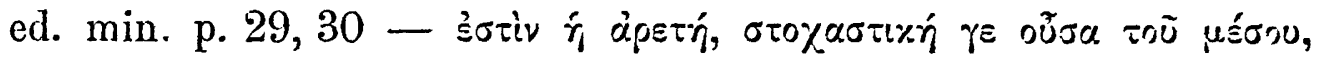

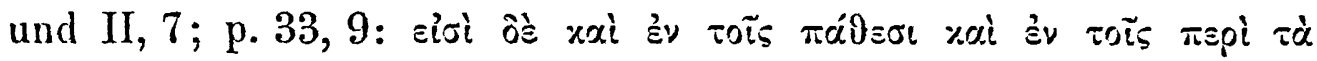

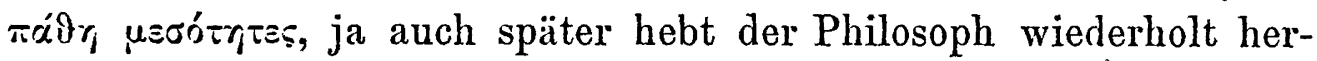

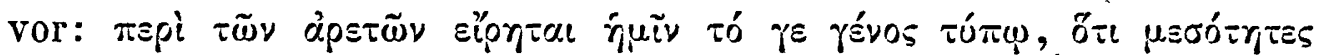

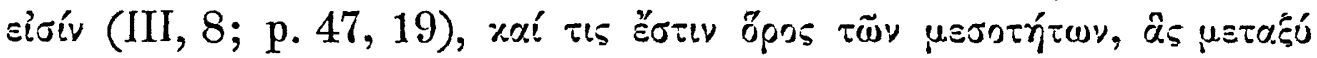

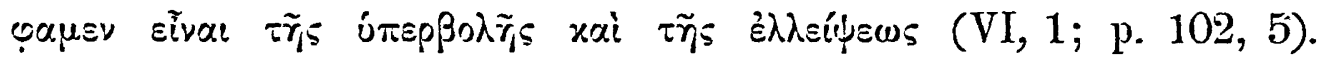
Musste ferner Apollinarios in seiner mit Athanasios und den Kappadociern im Wesentlichen übereinstimmenden Trinitätslehre, wie er sie auch in der von mir aus der fälschlich Justinus beigelegten

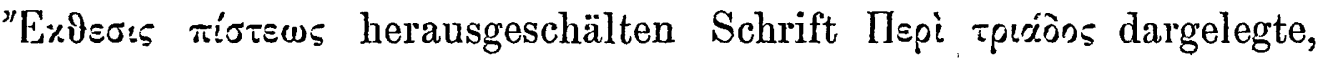
von platonischen Grundanschauungen ausgehen, so bediente er sich in derselben Schrift seinen Gegnern gegenüber aristotelischer Dialektik. Als besonders bezeichnend führe ich folgende Stelle an:

Mspi трсćiôos c. 14, p. $386 \mathrm{~B}$.

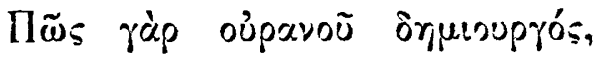

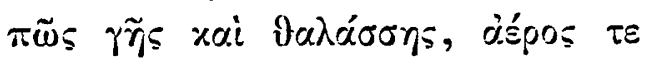

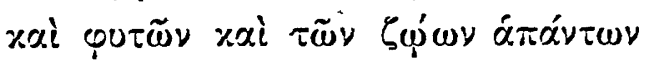

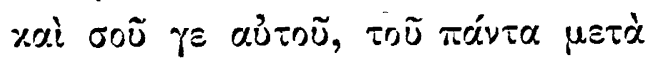

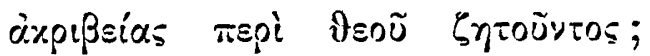

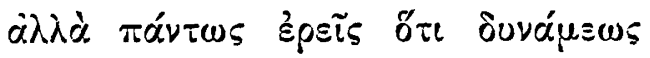

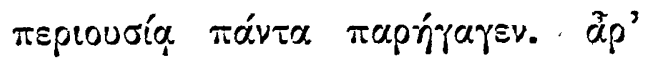

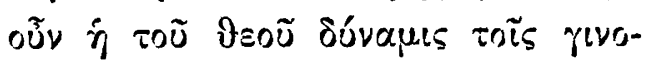

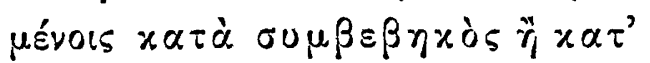

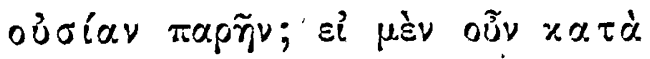

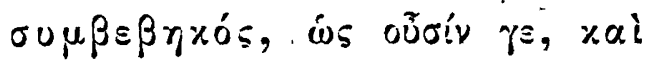

Arist. Phys. auscult. II, c. 6 .

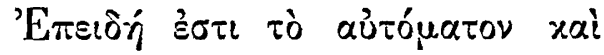

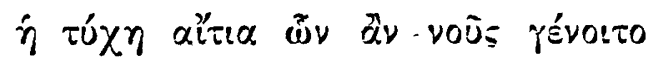

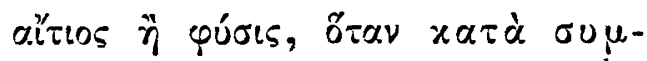

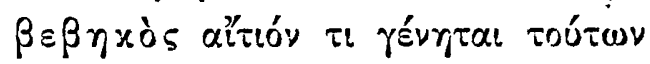

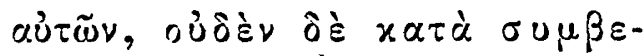

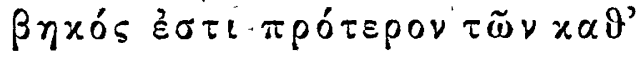

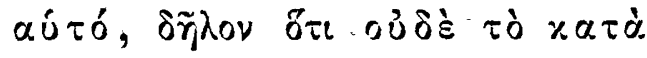

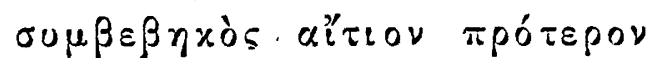

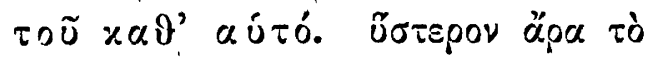

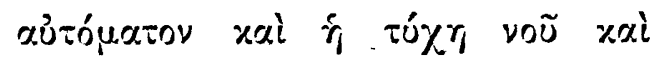

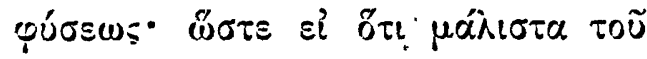




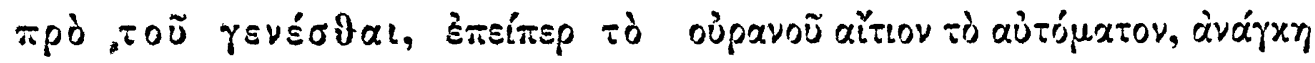

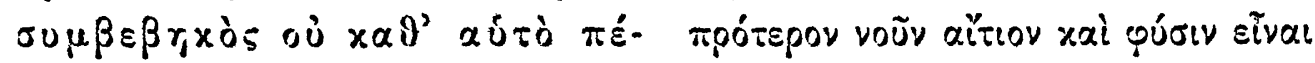

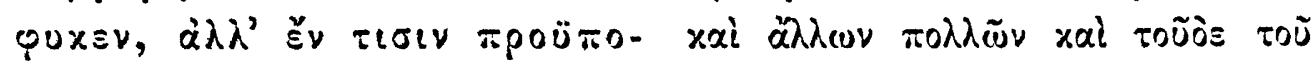

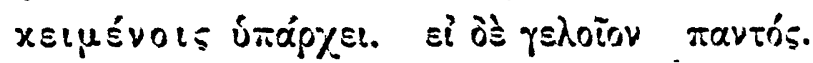

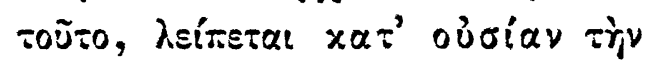

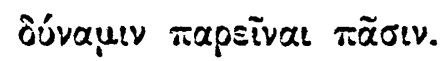

Wie sehr Apollinarios in aristotelischen Formen und Anschauungen dachte und lehrte, zeigt sich aber in keiner seiner Schriften so überzeugend, wie in seinen gegen die Arianer gerichteten Dialogen übor dio h. Dreieinigkeit, dio ich als von ihm herrührend und Jahrhunderte lang irrthümlich dem Athanasios oder Theodoretos (im V. Bande von Schulze's Ausgabe S. 916 ff.) beigelegt erwiesen habe ${ }^{16}$ ).

Im ersten Dialog handelt es sich um die Klarlegung des Cnterschiedes ron ojoía und ísészasts. Der Orthodoxe lehnt den

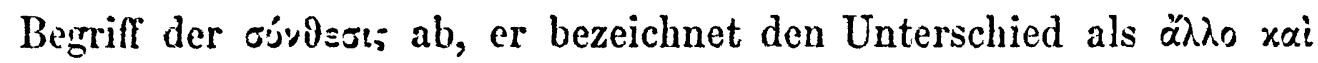
àj.r.

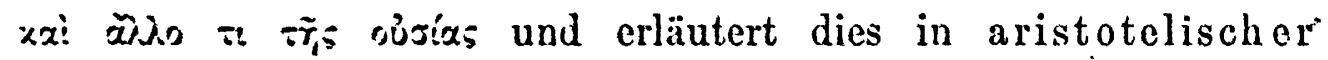

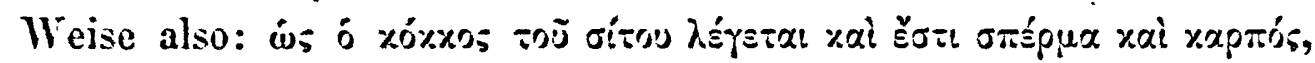

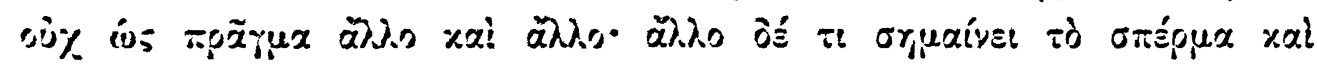

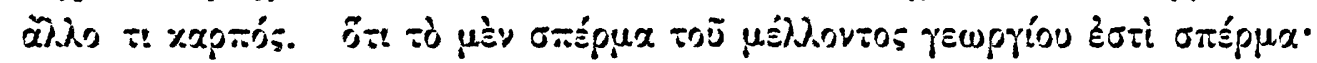

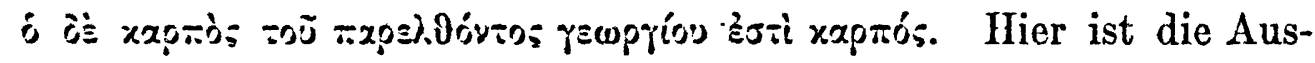
führung des Beispiels ganz entschieden eine solche, wie wir sie bei Aristoteles wiederholt treffen. Es fehlt nur die besondere Bezeichnung, welche dem Philosophen eigen ist, wonach er die Dinge

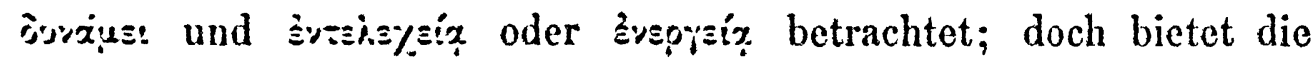
Stelle nicht die geringste Schwierigkeit, an sie jene aristotclische Unterscheidung heranzubringen. Aristotelisch sind dio ganzen folgenden Ausführungen (S. 934 und 935) über sỏsix und únistaбts, sachlich und sprachlich genau ïbereinstimmend mit dem, was A pollinarios in seiner soeben erwähnten Schrift IIspl tpex́osos (S. 373B und $374 \mathrm{~A}$ ) lehrt; aristotelisch die Erörterungen über $\theta=\delta_{0}=r_{5}$ und isis:xz:5 (S.933 und 939), vgl. besonders die S. 939 zu fin-

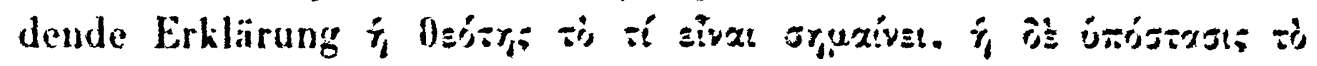

19) Theologische Studien und Kritiken, Jahrg. 1890, S. 137-171.

Arebiv t. Geeclichte d. Pbilonopties v. 


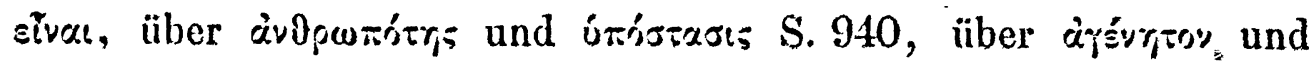
àrévvritov S. 941.

Gleichfalls echt aristotelisch ist die Ausführung im zweiten Dialog S. 980. Hier werden die beiden Begriffe हैะs und oủríx auf die Gotteslehre angewendet, und es, ist der Orthodoxe, der auf des

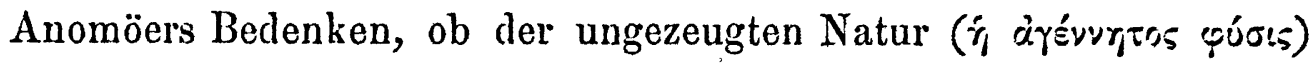
nicht vielmehr die Bezeichnung šsı als oủoía zukomme, die Streit-

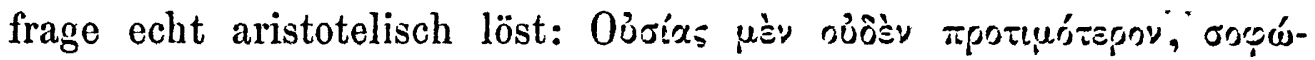

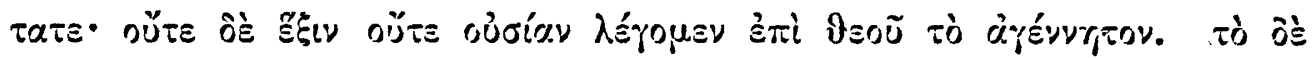

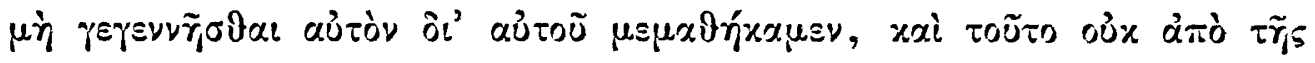

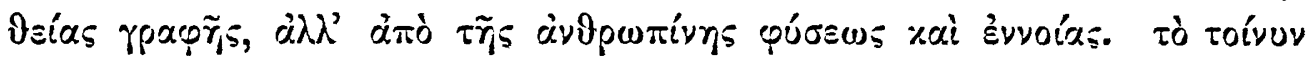

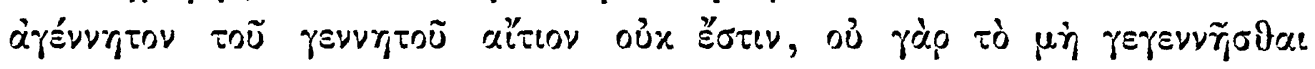

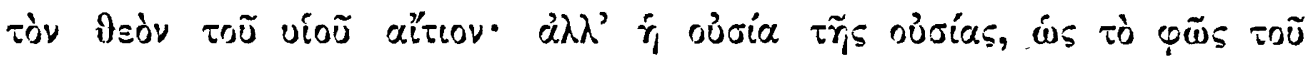

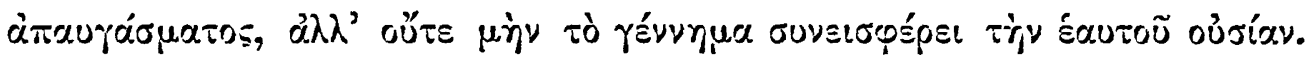

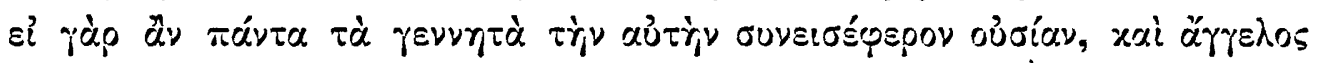

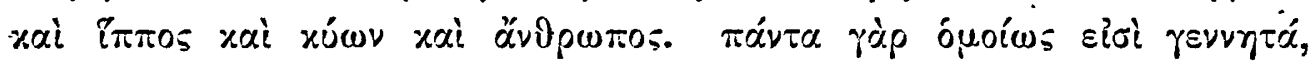

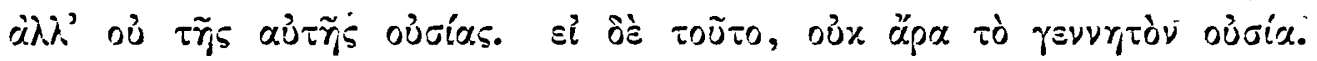

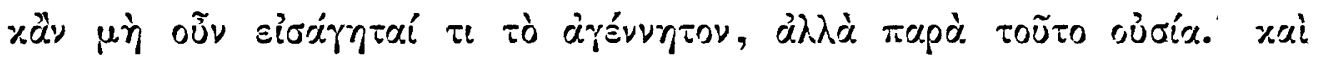

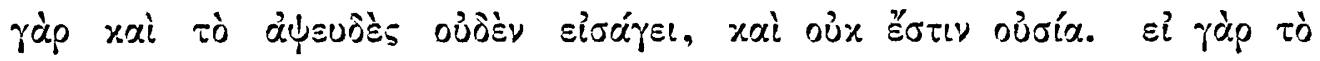

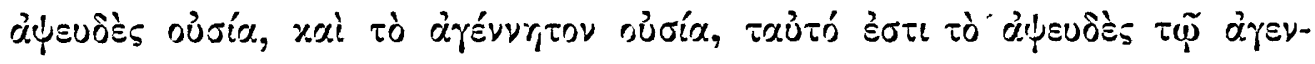
vír

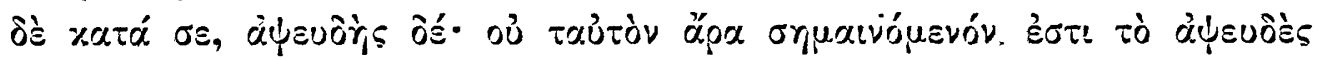

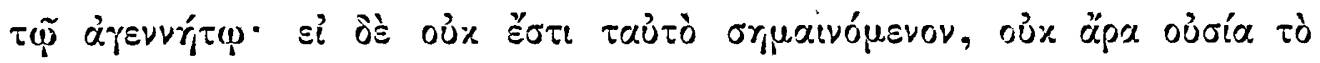

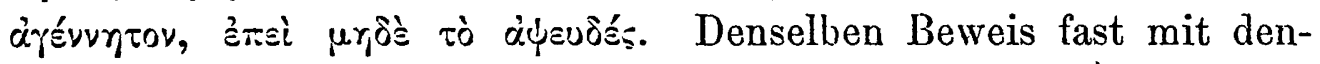
selben begrifflichen Mitteln des Aristoteles führt Apollinarios in seiner Schrift wider Eunomios (p. 284).

Genau ebenso hören wir endlich den Aristoteliker Apollinarios in dritten Dialoge reden. Der Orthodoxe hat (S. 1014) erklärt, der Mensch sei nach dem Ebenbilde Gottes erschaffen unter Mitwirkung des heiligen Geistes, da frägt er seinen Gegner, als dieser die Gottebenbildlichkeit allen Menschen zugesprochen wissen

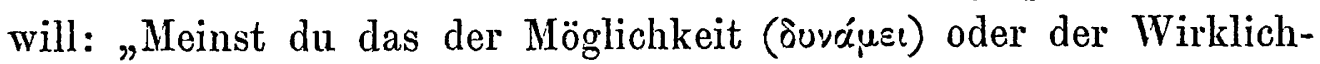

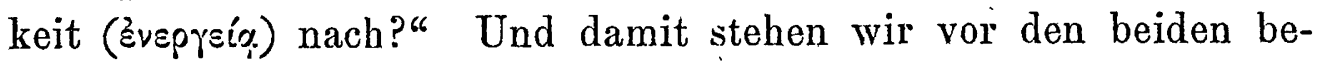
rühmten aristotelischen Hauptbegriffen, deren Bedeutung, Zusammenhang und Wechselbeziehung Trendelenburg in seinem Commentar zu Aristoteles' Büchern "Von der Seele“" (S. 295 bis.321 seiner 
Ausgabe) so lichtvoll und gründlich erlïutert hat. Der Gegnor weiss mit diesen begrifflichen Unterscheidungen nichts anzufangen;

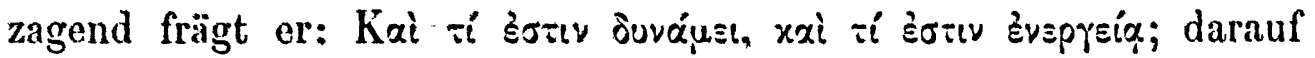

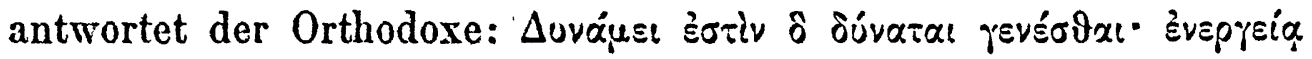

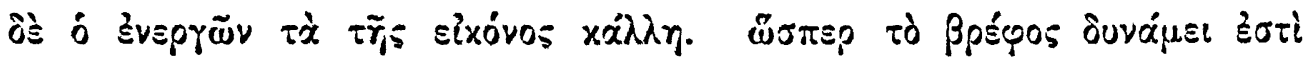

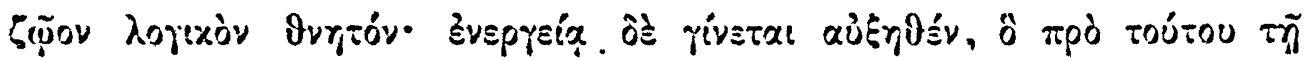

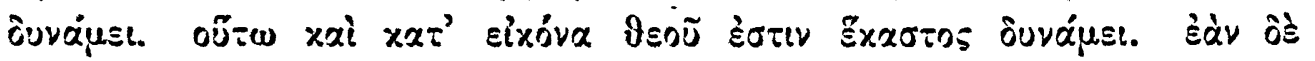

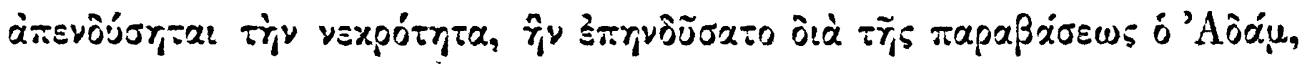

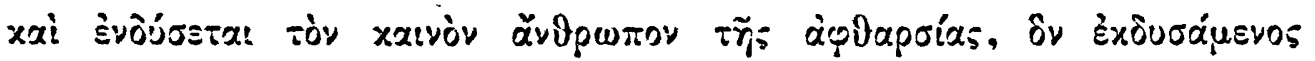

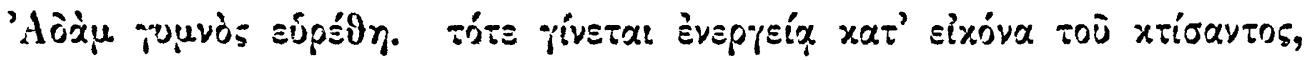

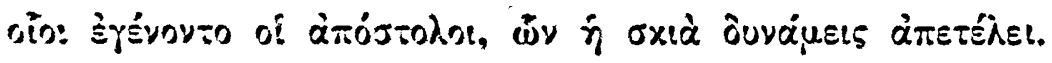

Ich denke durch diese meine Nachweisungen gezeigt zu haben, in wio herrorragend selbständiger Weise Apollinarios sich der Philosophie des Platon und A ristoteles für die Befestigung und Weiterentwickelung der Kirchenlehre bediente, und wie sehr Suidas mit seiner wahrscheinlich aus Philostorgios entlehnten Bemerkung Recht hat, wenn er über Apollinarios' Verdienste um die Dichtkunst hinaus das gerade rühmend hervorhob: $\pi 0 \lambda \lambda\left(\tilde{\mu} \pi \lambda \lambda^{i} \omega^{\prime}\right.$ xai $\varepsilon_{5}$

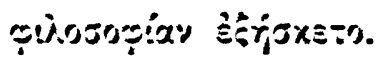




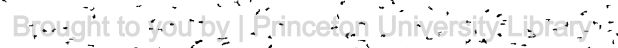

